\title{
Systematic Variation of Prosthetic Foot Spring Affects Center-of-Mass Mechanics and Metabolic Cost During Walking
}

\author{
Karl E. Zelik, Steven H. Collins, Peter G. Adamczyk, Ava D. Segal, Glenn K. Klute, David C. Morgenroth, \\ Michael E. Hahn, Michael S. Orendurff, Joseph M. Czerniecki, and Arthur D. Kuo
}

\begin{abstract}
Lower-limb amputees expend more energy to walk than non-amputees and have an elevated risk of secondary disabilities. Insufficient push-off by the prosthetic foot may be a contributing factor. We aimed to systematically study the effect of prosthetic foot mechanics on gait, to gain insight into fundamental prosthetic design principles. We varied a single parameter in isolation, the energy-storing spring in a prototype prosthetic foot, the controlled energy storage and return (CESR) foot, and observed the effect on gait. Subjects walked on the CESR foot with three different springs. We performed parallel studies on amputees and on non-amputees wearing prosthetic simulators. In both groups, spring characteristics similarly affected ankle and body center-ofmass (COM) mechanics and metabolic cost. Softer springs led to greater energy storage, energy return, and prosthetic limb COM push-off work. But metabolic energy expenditure was lowest with a spring of intermediate stiffness, suggesting biomechanical disadvantages to the softest spring despite its greater push-off. Disadvantages of the softest spring may include excessive heel displace-
\end{abstract}

Manuscript received October 31, 2010; revised May 02, 2011; accepted May 21, 2011. Date of publication June 23, 2011; date of current version August 10,2011 . This work was supported in part by the Department of Veterans Affairs, Rehabilitation Research and Development Service (A4372R), in part by the National Institutes of Health (R44HD055706), in part by the Department of Defense (DR081177), and in part by a National Science Foundation Graduate Research Fellowship.

This paper has supplementary downloadable material available at http://ieeexplore.iee.org, provided by the authors.

K. E. Zelik is with the Department of Mechanical Engineering, University of Michigan, Ann Arbor, MI 48109 USA.

S. H. Collins is with the Department of Mechanical Engineering, Carnegie Mellon University, Pittsburgh, PA 15213 USA.

P. G. Adamczyk is with the Intelligent Prosthetic Systems, Ann Arbor, MI 48109 USA.

A. D. Segal is with the Department of Veterans Affairs, RR\&D Center, Seattle, WA 98108 USA.

G. K. Klute is with the Department of Mechanical Engineering and the Department of Electrical Engineering, University of Washington, Seattle, WA 98108 USA, and also with the Department of Veterans Affairs, RR\&D Center, Seattle, WA 98108 USA

D. C. Morgenroth is with the Department of Veterans Affairs, RR\&D Center, Seattle, WA 98108 USA, and also with the Department of Rehabilitation Medicine, University of Washington, Seattle, WA 98108 USA

M. E. Hahn is with the Department of Veterans Affairs, RR\&D Center, Seattle, WA 98108 USA, and also with the Department of Mechanical Engineering, University of Washington, Seattle, WA 98108 USA.

M. S. Orendurff is with the Movement Science Laboratory, Texas Scottish Rite Hospital for Children, Dallas, TX 75219 USA.

J. M. Czerniecki is with the Department of Rehabilitation Medicine, University of Washington, Seattle, WA 98108 USA, and also with the Department of Veterans Affairs, RR\&D Center, Seattle, WA 98108 USA

A. D. Kuo is with the Department of Mechanical Engineering and the Department of Biomedical Engineering, University of Michigan, Ann Arbor, MI 48109 USA.

Color versions of one or more of the figures in this paper are available online at http://ieeexplore.ieee.org.

Digital Object Identifier 10.1109/TNSRE.2011.2159018 ments and COM collision losses. We also observed some differences in joint kinetics between amputees and non-amputees walking on the prototype foot. During prosthetic push-off, amputees exhibited reduced energy transfer from the prosthesis to the COM along with increased hip work, perhaps due to greater energy dissipation at the knee. Nevertheless, the results indicate that spring compliance can contribute to push-off, but with biomechanical trade-offs that limit the degree to which greater push-off might improve walking economy.

Index Terms-Amputee gait, ankle push-off, prosthetic feet, prosthetic simulator.

\section{INTRODUCTION}

$\mathbf{P}$ ROSTHETIC foot technology has undergone substantial transformation over recent decades, most notably with the introduction of elastic materials and mechanisms. Despite a wide variety of implementations, there remain disadvantages to amputee gait that lead to greater fatigue, secondary disabilities, and reduced mobility [1]-[7]. Further improvement of prostheses is hindered by a lack of quantitative principles for design and prescription. By systematically studying specific mechanical parameters and quantifying the effects, it might be possible to extract design principles that could help to improve comfort and reduce fatigue in amputee gait.

Ankle push-off mechanics may be an important factor affecting amputee mobility and walking economy. Healthy nonamputees typically perform a large burst of positive ankle work during the end of the stance phase of gait, whereas amputees wearing conventional prosthetic feet exhibit much less ankle/ foot push-off [8], and less center-of-mass (COM) push-off work [9]. Reduced push-off may lead to greater collisional energy losses of the leading limb after heelstrike [10]-[12], and may therefore require additional muscle work from other joints to compensate. Observed differences in joint kinetics between amputees and non-amputees [13] may reflect these and other compensatory actions and perhaps explain some of the increase in metabolic cost [14].

Push-off can be affected by elastic features of prosthetic feet. Dynamic elastic response feet [15], [16] incorporate passive spring-like components that return elastic energy during unloading of the foot and may thus contribute to push-off. Other devices attempt to augment push-off more directly using active control. For example, a prosthetic foot prototype, the controlled energy storage and return (CESR) foot, captures 
energy from the heelstrike collision and releases it elastically at push-off [17]. Another prototype, the MIT powered ankle/foot prosthesis, drives push-off both actively and elastically [18]. Such devices raise the question of what compliance should be specified in the design process.

Although various prosthetic foot designs have been evaluated against each other in comparison studies (e.g., [1], [19]-[21]), it is difficult to extract specific design principles regarding mechanical compliance (e.g., [22]). This is in part because even superficially similar feet (e.g., Seattle Lightfoot2 and FlexFoot, both categorized as dynamic elastic response feet) vary not only in compliance, but also in geometry, mass, alignment, and material type. These differences confound the ability to directly attribute differences in measured outcomes to specific design characteristics.

An alternative to comparing different brands or designs of prosthetic feet is to test one foot while systematically varying a single isolated parameter. Systematic manipulation of prosthetic parameters have only been performed in a few previous studies (e.g., [23], [24]), but could lead to greater insight regarding design principles for improving amputee gait, particularly those related to ankle push-off mechanics. This is especially the case for more complex prostheses that employ active mechanisms which supplement or control passive compliance. Of particular interest in these devices is the selection of the passive compliant components that contribute to push-off and govern much of the mechanical behavior of the ankle/foot. Systematic studies would allow for direct attribution of altered gait mechanics to a specific prosthetic foot parameter.

The potential efficacy of parametric studies is illustrated by previous experiments performed on non-amputee subjects wearing prosthetic simulator boots. Simulator boots immobilize the ankle and allow for attachment of a prosthesis beneath the foot [25]. These prosthetic simulators have been used to systematically study how convex foot bottom shape can affect energy economy [12] and how prosthetic alignment relates to rollover shape [26]. Of course, even with ankle fixation, there remain numerous differences with amputees, including interface, musculature, and sensory information. Non-amputees walking on prosthesis simulators are not intended to be a model of amputee gait, but may nonetheless represent one way individuals walk in the absence of an articulating ankle joint and the associated musculature. Comparing amputees and non-amputees walking on the same prosthetic foot may therefore provide additional insight into how and why amputation affects gait.

The main purpose of this study was to systematically vary a single component within a prosthetic foot and experimentally measure the resulting effect on gait mechanics and metabolic energy expenditure. We studied amputee subjects to test how spring characteristics in the CESR foot affected their gait. We also studied non-amputees with prosthetic simulator boots to test for general effects that apply to walking without an active ankle, even without amputation. We measured push-off work and other variables as a function of systematically varied stiffness of an energy-recycling spring component within a prototype CESR prosthesis. The CESR spring strongly affects ankle push-off work and is therefore amenable to systematic variation. We then observed how this altered prosthetic ankle work

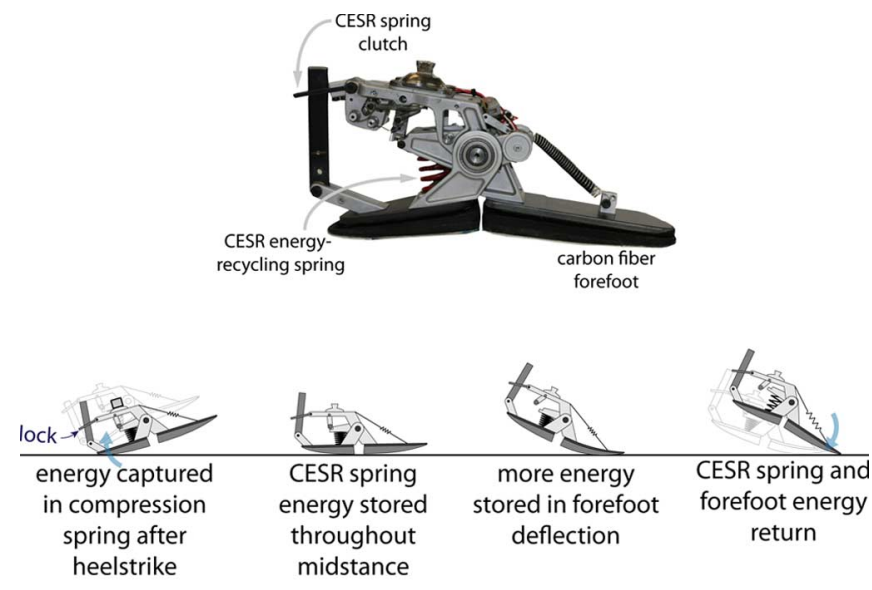

Fig. 1. CESR prosthesis prototype. The CESR foot stores energy in a compression spring after heelstrike, locks the spring energy into place with a clutch and then returns the energy during terminal stance in the form of plantarflexion push-off work. This energy recycling foot provides improved energy return as compared to conventional passive prostheses [17].

affected gait. We predicted that the energy returned for push-off would be proportional to the amount stored in the spring during the foot's collision with ground, with that amount in turn dependent on spring stiffness. An improved understanding of specific prosthetic foot parameters and design principles could offer important insights that may enable improvement in amputee gait.

\section{METHODS}

We studied level-ground walking mechanics and metabolic energy expenditure of unilateral, transtibial amputees $(N=5)$ walking on the CESR foot (Fig. 1), while varying its energy-recycling spring between three stiffness levels. We also performed a closely paralleled study on non-amputees wearing simulator boots $(N=11)$. We tested the effect of stiffness on work performed on the COM, joint work measures, and oxygen consumption. We also measured subjects walking on a representative conventional prosthesis, the Seattle LightFoot2 [27] (Seattle Systems, Poulsbo, WA), as a qualitative control. To reduce possible confounds from prosthesis weight we added mass to the conventional foot to match the CESR foot $(1.37 \mathrm{~kg})$. Prior to the study, all subjects provided their informed consent according to Institutional Review Board procedures. Methods are outlined below and further details are reported in the supplementary material.

The CESR prototype is designed to recycle energy that is largely dissipated in walking collisions [17]. It stores energy elastically at the heel during loading of the foot in early stance (termed collision), locks the spring throughout mid-stance with a one-way clutch, and releases the spring energy near terminal stance in the form of plantarflexion push-off work (Fig. 1). Unlike conventional passive prosthetic feet that store and return elastic energy in the heel only during early stance, heel energy stored in the CESR can be controlled and released to help increase prosthetic limb push-off during terminal stance. The amount of CESR energy return was expected to be dependent on the energy-recycling spring properties varied in this study. The CESR foot is not intended to represent conventional prosthetic 
feet, but is a useful tool for studying the response of amputee gait to push-off related parametric variation in a prosthesis.

All subjects were tested while walking on three different CESR energy-recycling springs. Spring specifications ${ }^{1}$ were selected in an attempt to systematically vary energy storage and return in the prosthetic foot. These springs were termed according to their stiffness as hard (stiffest), medium, and soft-PC (softest, pre-compressed) springs. We found the soft spring to have insufficient stiffness to prevent the heel component from reaching its maximum displacement limit during walking. We therefore found it helpful to pre-compress (PC) the soft spring beneath the heel, ${ }^{1}$ so that the soft-PC spring exhibited increased spring force for a given heel displacement and thus higher energy storage capacity. ${ }^{2}$ Amputees walked on a slightly less stiff hard spring compared to non-amputees due to comfort-related issues (see supplementary material). During testing, Amputee subjects walked at $1.14 \mathrm{~m} / \mathrm{s}$ and non-amputees at $1.25 \mathrm{~m} / \mathrm{s}$ to approximate typical self-selected walking speeds. We measured ground reaction forces, full-body kinematics, and oxygen consumption and carbon dioxide production. These tests were conducted on the three springs, applied in random order. Walking on a conventional prosthesis was also collected as a control condition. Non-amputees wore the prostheses unilaterally on a prosthesis simulator boot, with a lift shoe on the contralateral foot, and also performed an additional Shod condition with normal street shoes on both feet. All amputee prosthesis alignments were performed by the same experienced prosthetist. Typical clinical practice would involve realigning the foot for each new component in order to maximize the functional benefit to the user. However, in order to preserve the systematic nature of our study, springs were exchanged without disturbing prosthetic alignment, thus avoiding effects due to variation of an addition parameter. CESR alignments were performed with the medium spring. Prior to the testing day, subjects were given a brief acclimation period, in which they walked on the treadmill and overground while wearing the CESR prototype in each of the three spring configurations.

We compared mechanical and metabolic results across spring conditions, and looked for similarities and differences in trends between amputee and non-amputee subjects. We computed the following mechanical estimates: 1) rate of work performed on the body center-of-mass (COM) by the individual limbs [28], 2) ankle/foot power using an inter-segmental energy balance calculation between the ankle/foot and rest of body [29]-[31], and 3) knee and hip joint power using standard inverse dynamics (e.g., [32]). Ankle/foot power was estimated using the inter-segmental power method rather than traditional inverse dynamics because elastic portions of prosthetic feet are difficult to model as rigid bodies [31]. The inter-segmental approach treats the entire foot and ankle as a deformable body and measures the instantaneous forces and velocities of its contact with the ground and leg to estimate mechanical power due to both rotation and translation. We additionally used displacement sensors on the CESR foot to estimate elastic energy storage in the energy-recycling spring. We computed summary work

\footnotetext{
${ }^{1}$ See Table S1 in supplementary downloadable material.
}

${ }^{2}$ See Figure S6 in supplementary downloadable material.
Table I

ENERGY CAPTURED BY CESR SPRING (J)

\begin{tabular}{l|l|l} 
& \multicolumn{1}{|c|}{ Amputees } & \multicolumn{1}{c}{ Non-Amputees } \\
\hline Hard & $-6.5(1.0)^{\mathrm{a}}$ & $-6.2(1.3)^{\mathrm{c}, \mathrm{d}}$ \\
Medium & $-8.1(2.8)^{\mathrm{b}}$ & $-8.9(1.2)^{\mathrm{c}, \mathrm{e}}$ \\
Soft-PC & $-16.6(1.0)^{\mathrm{a}, \mathrm{b}}$ & $-13.3(3.3)^{\mathrm{d}, \mathrm{e}}$ \\
\hline P-values & ${ }^{\mathrm{a}} 6.9 \mathrm{e}-4$ & ${ }^{\mathrm{c}} 2.9 \mathrm{e}-5$ \\
(alpha=0.017) & ${ }^{\mathrm{b}} 0.0077$ & ${ }^{\mathrm{d}} 4.1 \mathrm{e}-6$ \\
& & ${ }^{\mathrm{e}} 6.9 \mathrm{e}-5$
\end{tabular}

measures by integrating the power estimates over phases of the gait cycle — collision, rebound, preload, push-off, swing - as defined by fluctuating regions of positive and negative COM work rate [33], [34]. We also estimated metabolic energy consumption using indirect calorimetry [35]. Within a subject group and across spring conditions, statistical analysis was performed using a repeat measures ANOVA with a Holm-Sidak correction. No statistical tests were performed comparing the conventional foot to any other condition. Mechanical and metabolic measures for amputee versus non-amputee subjects were not tested statistically either due to differences in experimental protocols, such as walking speed, average age, and leg length due to the simulator boot height (see complete methodological details in supplementary material). Inter-group similarities and differences may, however, still provide useful insight and therefore are compared qualitatively in terms of work magnitudes and trends across spring conditions.

Mechanical work measures are summarized in Tables I-IV. Superscript letters indicate statistically significant differences between the two quantities bearing the same letter.

\section{RESULTS}

The results are reported separately for amputees and nonamputees, followed by qualitative observations comparing both groups. In all figures and tables, the results represent the means and standard deviations computed across all subjects.

\section{A. Amputees}

Prosthetic foot mechanics varied with spring stiffness (Fig. 2). Greater energy storage in the spring during collision translated into more work performed in the subsequent ankle/foot push-off. The amount of energy stored and returned by the prosthesis increased with decreasing stiffness. The soft-PC energy-recycling spring stored the most energy (16.6 (1.0) J), followed by the medium spring (8.1 (2.8) J), and then the hard spring (6.5 (1.0) J; Table I). Energy stored in the forefoot keel was of similar magnitude for all CESR conditions, about $8.5 \mathrm{~J}$ for amputees, based on ankle/foot power estimates (see Fig. 2(a), negative work during $40 \%-80 \%$ of stance phase). Therefore, prosthetic energy return (from both the energy-recycling spring and the elastic forefoot) followed the same trend as energy storage, with soft-PC performing the most prosthetic push-off work (24.9 (1.5) J), medium in the middle (20.0 (1.3) J), and the hard spring performing the least (18.8 (1.9) J). The soft-PC spring stored and returned significantly more energy than the medium and hard springs $(\mathrm{P}<0.008$; Table II). On average, the medium spring stored and returned 
Table II

ANKLE PUSH-OFF (J)

\begin{tabular}{|c|c|c|c|c|}
\hline & \multicolumn{2}{|c|}{ Prosthetic Limb } & \multicolumn{2}{|c|}{ Intact Limb } \\
\hline & Amputees & Non-Amputees & Amputees & Non-Amputees \\
\hline Hard & $18.8(1.9)^{\mathrm{h}}$ & $22.1(2.7)^{\mathrm{j}, \mathrm{k}}$ & $18.3(2.6)$ & $16.5(4.0)$ \\
\hline Medium & $20.0(1.3)^{\mathrm{i}}$ & $24.1(2.4)^{\mathrm{j}, \mathrm{I}}$ & $17.8(2.2)$ & $17.6(4.7)$ \\
\hline Soft-PC & $24.9(1.5)^{\mathrm{h}, \mathrm{i}}$ & $26.7(3.5)^{\mathrm{k}, \mathrm{l}}$ & $19.1(3.0)$ & $17.5(5.2)$ \\
\hline Conventional $^{\prime}$ & $12.2(2.8)$ & $10.2(1.8)$ & $20.4(3.1)$ & $15.1(3.7)$ \\
\hline Shod $d^{\dagger}$ & N/A & N/A & N/A & $16.8(4.4)$ \\
\hline $\begin{array}{l}\text { P-values } \\
\text { (alpha }=0.017 \text { ) }\end{array}$ & $\begin{array}{l}{ }^{\mathrm{h}} 0.0048 \\
\mathrm{i} 0.0012\end{array}$ & $\begin{array}{l}{ }^{\mathrm{j}} 1.4 \mathrm{e}-4 \\
{ }^{\mathrm{k}} 1.1 \mathrm{e}-5 \\
{ }^{\mathrm{l}} 0.0076\end{array}$ & All $>0.18$ & All $>0.051$ \\
\hline
\end{tabular}
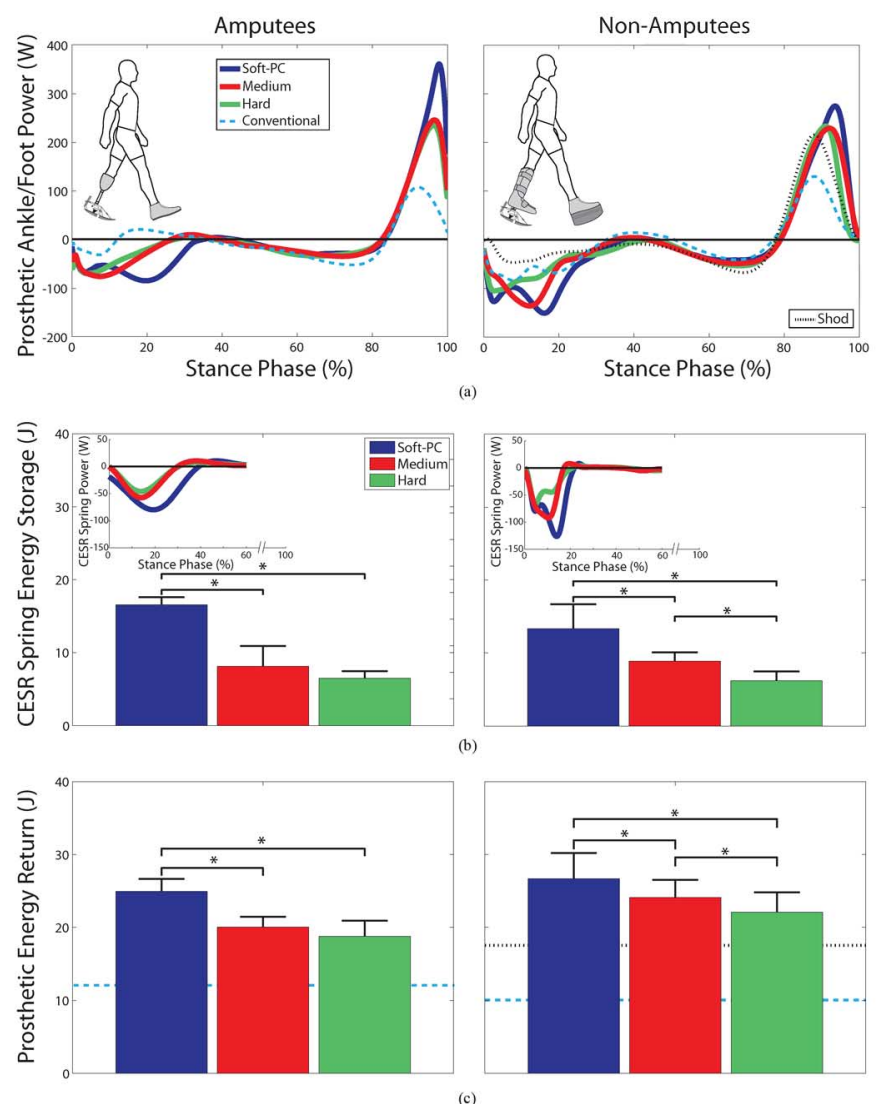

Fig. 2. Prosthesis power, energy storage and return. Average (a) ankle/foot power, (b) CESR spring energy storage and (c) prosthetic energy return are shown for amputees (left column) and non-amputees wearing simulator boots (right column). The (a) ankle/foot power estimates all energy flow into and out of the foot, and the inset in (b) CESR spring power, represents energy storage contributions of just the spring. Power is plotted across stance phase from heelstrike to toe-off. Results from a Conventional passive prosthesis (dashed line) and typical shod non-amputee gait (dotted line) are provided for reference. Prosthetic ankle/foot mechanics varied with spring stiffness in both amputees and non-amputees: the softest, pre-compressed (soft-PC) spring stored and returned the most energy, while the stiffest (hard) spring stored and returned the least. In all figures, the results represent the means and standard deviations computed across all subjects.

more energy than the hard spring, but this difference did not achieve statistical significance in amputees $(P=0.17)$.

Spring stiffness also affected COM mechanics (Fig. 3). Push-off work performed on the COM followed the same trend as ankle/foot work, tending to increase with decreasing spring stiffness. The soft-PC spring resulted in the most COM push-off work (17.7 (2.6) J), followed by the medium spring (15.3 (1.8) J). Similar to ankle push-off, COM push-off was slightly lower with the hard spring (14.9 (2.3) J) than medium, but the difference again did not reach statistical significance. In amputees, varying from the hard to soft-PC spring led to a $32 \%$ increase in ankle/foot push-off work and a 19\% increase in COM push-off work.

Other mechanical measures showed little variation with spring stiffness. Firstly, spring choice had little effect on COM work rate of the intact limb (Fig. 3). For instance, intact limb $\mathrm{COM}$ work measures were not found to differ across spring conditions during collision $(\mathrm{P}>0.46)$ or push-off $(\mathrm{P}>0.09)$ phases of gait (Tables III and IV). Secondly, prosthetic limb knee and hip kinetics ${ }^{3}$ (Fig. 5) and intact limb ankle, knee and hip kinetics ${ }^{4}$ exhibited little change with spring stiffness.

Metabolic energy expenditure was also affected by spring choice (Fig. 4). On average, the medium spring yielded the lowest net metabolic rate, $4 \%$ lower than the soft-PC spring and $5 \%$ lower than the hard spring. This result reached statistical significance for the medium versus soft-PC comparison in amputees $(P=0.0025)$. A strong trend was also observed in comparing medium versus hard, but it did not reach statistical significance $(\mathrm{P}=0.076)$.

Amputee gait kinetics while walking on the Conventional foot appeared qualitatively consistent with prior literature on below-knee amputee gait (e.g., [13]).

\section{B. Non-Amputees}

CESR energy storage and return increased with softening spring stiffness in non-amputees (Fig. 2). The soft-PC spring stored the most energy (13.3 (3.3) J), followed by the medium (8.9 (1.2) J) and hard (6.2 (1.3) J) springs (all differences statistically significant, $\mathrm{P}<7 e-5$; Table I). Prosthetic ankle push-off work was highest with the soft-PC spring (26.7 (3.5) J), followed by medium (24.1 (2.4) J) and hard (22.1 (2.7) J) springs (all differences statistically significant, $\mathrm{P}<0.008$; Table II).

In non-amputees, prosthetic limb COM push-off work also tended to increase with softening spring stiffness [Fig. 3(b)]. COM push-off work was 28.4 (3.0) J, 26.9 (3.0) J, and 25.8 (2.6) $\mathrm{J}$ for soft-PC, medium, and hard springs, respectively.

\footnotetext{
${ }^{3}$ See Figures S3 and S5 in supplementary downloadable material

${ }^{4}$ See Figures S2 and S4 in supplementary downloadable material
} 
Table III

COM COLLISION WORK (J)

\begin{tabular}{|c|c|c|c|c|}
\hline & \multicolumn{2}{|c|}{ Prosthetic Limb } & \multicolumn{2}{|c|}{ Intact Limb } \\
\hline & Amputees & Non-Amputees & Amputees & Non-Amputees \\
\hline Hard & $-7.7(3.4)$ & $-18.4(5.2)^{f}$ & $-3.0(1.8)$ & $-14.4(4.6)$ \\
\hline Medium & $-8.3(5.6)$ & $-18.5(3.5)^{\mathrm{g}}$ & $-3.3(2.0)$ & $-12.9(4.7)$ \\
\hline Soft-PC & $-10.0(7.4)$ & $-22.7(5.2)^{\mathrm{f}, \mathrm{g}}$ & $-3.1(1.8)$ & $-12.8(3.9)$ \\
\hline$\overline{\text { Conventional }} l^{\dagger}$ & $-7.6(3.8)$ & $-17.3(3.5)$ & $-13.0(5.9)$ & $-12.6(4.9)$ \\
\hline Shod $d^{t}$ & $N / A$ & $N / A$ & $N / A$ & $-10.4(3.5)$ \\
\hline $\begin{array}{l}\text { P-values } \\
\text { (alpha }=0.017 \text { ) }\end{array}$ & All $>0.16$ & $\begin{array}{l}{ }^{\mathrm{f}} 1.4 \mathrm{e}-4 \\
\mathrm{~g}_{1} .6 \mathrm{e}-4\end{array}$ & All $>0.46$ & All $>0.029$ \\
\hline
\end{tabular}
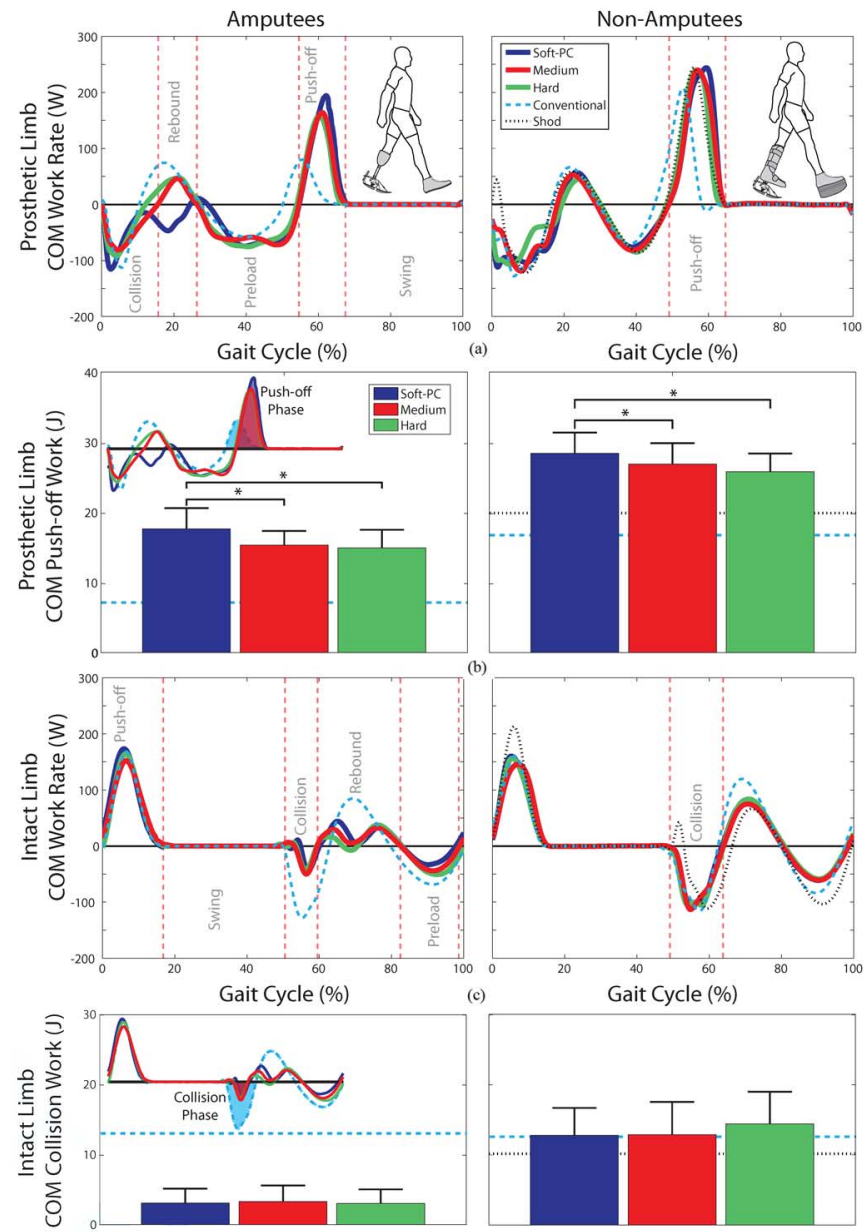

(d)

Fig. 3. COM work rate, prosthetic limb push-off and intact limb collision work. Average COM work rate for (a) prosthetic and (c) intact limbs are plotted across a full gait cycle (beginning with prosthetic heelstrike) for amputees (left column) and non-amputees (right column). Phases of gait—collision, rebound, preload, push-off, swing - are defined for each limb based on alternating regions of positive and negative COM work, and approximate regions are shown for the medium spring by vertical lines. The (b) prosthetic limb Push-off work and (d) intact limb collision work were integrated from the shaded phases shown, which were defined independently for each subject and condition. Softer spring stiffness led to increased prosthetic limb COM push-off, but stiffness did not have a significant effect on intact limb collision work. Also, amputees appeared to perform less prosthetic limb COM push-off work and less intact limb COM collision work than non-amputees. Reference lines are shown for walking on a Conventional prosthesis (dashed line) and in street shoes (dotted line) for non-amputees.

Soft-PC versus medium $(\mathrm{P}=5 \mathrm{e}-4)$ and soft-PC versus hard $(\mathrm{P}=7 \mathrm{e}-4)$ comparisons showed statistically significant differences. In non-amputees, varying spring properties yielded a $21 \%$ increase in ankle/foot push-off work and a $10 \%$ increase in COM push-off work.

Some other mechanical measures showed little variation across the gait cycle as a function of spring choice. These included intact limb sagittal plane joint kinetics and kinematics, 5 intact limb COM work rate (Fig. 3), and prosthetic limb sagittal plane knee and hip kinetics ${ }^{6}$ (Fig. 5).

In non-amputees, the average metabolic rate was lowest while walking on the medium spring by $7 \%-8 \%$ as compared to the other spring conditions (Fig. 4). This result was statistically significant for the medium versus hard comparison $(\mathrm{P}=0.0045)$, but did not quite reach significance for the medium versus soft-PC comparison $(\mathrm{P}=0.063)$.

\section{Amputees Versus Non-Amputees}

We observed similar trends in gait mechanics and metabolic cost as a function of spring stiffness for both amputee and nonamputee subjects walking on the CESR foot. In both groups, varying spring stiffness led to similar systematic changes in foot function, joint kinetics and whole-body gait mechanics. Softer springs led to more energy storage and return in the CESR foot, which in turn led to more push-off work performed by the prosthetic ankle and more push-off work performed on the body COM. Intact limb ankle, knee and hip kinetics and prosthetic limb knee and hip kinetics showed little variation as a function of CESR spring stiffness. Additionally, metabolic energy expenditure was $4 \%-8 \%$ lower for both groups walking with the medium spring than the soft-PC or hard springs.

We observed similar mechanical function of the CESR foot in both groups, as indicated by similar magnitudes of energy storage and return performed by the prosthesis (Fig. 2). Prosthetic energy storage for the hard, medium, and soft-PC springs varied from about 7-17 $\mathrm{J}$ in amputees and from about 6-13 J in non-amputees. Prosthetic ankle push-off work varied from about $19-25 \mathrm{~J}$ in amputees and from about 22-27 J in non-amputees.

However, between groups there were also some substantial differences in COM and joint work magnitudes, specifically during the push-off phase of gait. Prosthetic limb COM push-off work appeared substantially lower (by about $10 \mathrm{~J}$ ) in amputees (15-18 J) than non-amputees (26-28 J) walking on the CESR

\footnotetext{
${ }^{5}$ See Figures $\mathrm{S} 2$ and $\mathrm{S} 3$ in supplementary downloadable material

${ }^{6}$ See Figures S4 and S5 in supplementary downloadable material
} 
Table IV

COM PUSH-OFF WORK (J)

\begin{tabular}{|c|c|c|c|c|}
\hline & \multicolumn{2}{|c|}{ Prosthetic Limb } & \multicolumn{2}{|c|}{ Intact Limb } \\
\hline & Amputees & Non-Amputees & Amputees & Non-Amputees \\
\hline Hard & $14.9(2.3)^{\mathrm{m}}$ & $25.8(2.6)^{0}$ & $16.9(4.2)$ & $18.2(5.7)$ \\
\hline Medium & $15.3(1.8)^{\mathrm{m}}$ & $26.9(3.0)^{p}$ & $17.4(4.8)$ & $18.9(5.8)$ \\
\hline Soft-PC & $17.7(2.6)^{\mathrm{m}, \mathrm{n}}$ & $28.4(3.0)^{\mathrm{o}, \mathrm{p}}$ & $19.1(5.2)$ & $18.9(7.1)$ \\
\hline Conventional $^{t^{\prime}}$ & $7.3(1.4)$ & $17.1(2.9)$ & $15.3(3.1)$ & $16.9(5.1)$ \\
\hline$\underline{S h o d}^{\dagger}$ & $N / A$ & $N / A$ & $N / A$ & $16.7(4.9)$ \\
\hline $\begin{array}{l}\text { P-values } \\
(\text { alpha }=0.017)\end{array}$ & $\begin{array}{l}{ }^{\mathrm{m}} 0.0028 \\
{ }^{\mathrm{n}} 0.014\end{array}$ & $\begin{array}{l}{ }^{\circ} 4.7 \mathrm{e}-4 \\
{ }^{\mathrm{p}} 6.9 \mathrm{e}-4\end{array}$ & All $>0.095$ & All $>0.2$ \\
\hline
\end{tabular}

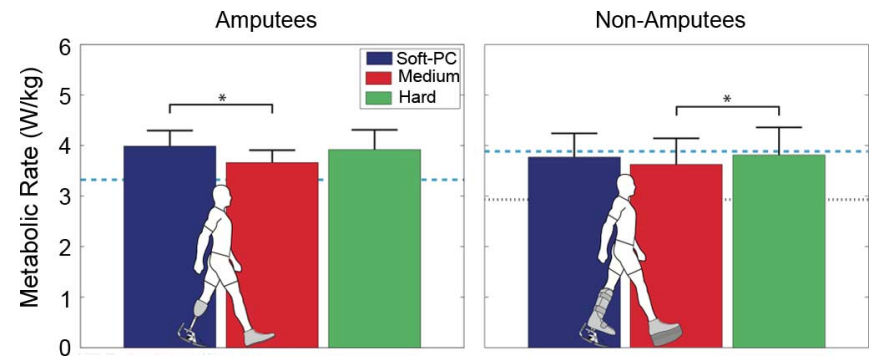

Fig. 4. Average metabolic rate for amputees (left) and non-amputees (right). Results for a Conventional passive prosthesis (dashed line) and shod non-amputee gait (dotted line) are shown for reference. We observed mixed metabolic results that were qualitatively similar in amputee and non-amputee subjects across spring conditions. The medium spring was significantly lower than soft-PC spring in amputees, and lower than the hard spring in non-amputees subjects. Although metabolic energy expenditures were found to be of similar magnitudes in both amputees and non-amputees, we consider this coincidental and attribute no special significance to the absolute comparison. There was no reason a priori to expect similar energetic magnitudes given differences between groups and experimental protocols. Qualitatively, amputee subjects tended to expend more energy walking on the CESR foot than on the Conventional prosthesis, whereas non-amputees tended to expend less energy while walking on the CESR.

foot [Fig. 3(b)]. A similar difference was observed between the two groups with the Conventional prosthesis (7 versus 17 J). Prosthetic limb knee and hip kinetics also differed between groups. Inverse dynamics estimates indicated that amputees performed substantial prosthetic-side negative knee work (about $11 \mathrm{~J}$ ) during push-off [Fig. 5(b)]. In contrast, non-amputees exhibited prosthetic-side positive knee work (about $1.5 \mathrm{~J}$ ) during push-off. Amputees performed about five times more prosthetic side hip work during push-off (approximately $13 \mathrm{~J}$ versus $2.5 \mathrm{~J}$ ) compared to non-amputees [Fig. 5(d)]. With the Conventional prosthesis, we made the same observation: prosthetic side knee and hip push-off work magnitudes were substantially larger in amputees than non-amputees.

\section{Discussion}

We studied how systematic changes in energy-storing spring properties affected amputees and non-amputees walking on a CESR prosthetic foot prototype. We found that several features of gait changed as a function of spring stiffness, including COM push-off and collision mechanics, and that amputee and non-amputee groups both shared many similar trends in mechanical and metabolic measures. Softer springs stored and returned more energy, leading to higher prosthetic
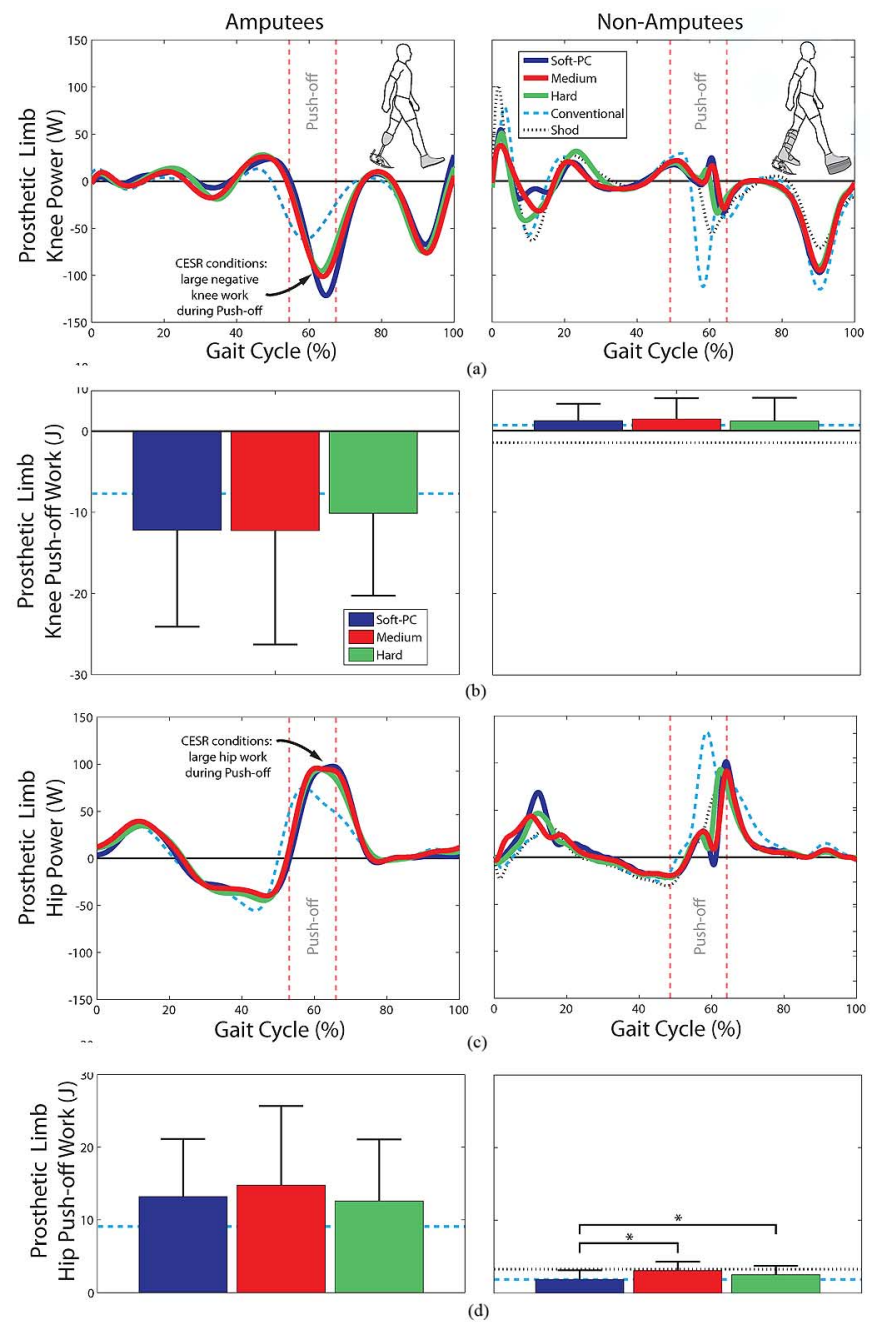

Fig. 5. Prosthetic limb knee and hip powers, work during push-off phase. Average prosthetic (a) knee and (c) hip are plotted across the full gait cycle (beginning with prosthetic heelstrike) for amputees (left) and non-amputees (right). The prosthetic limb (b) knee work and (d) hip work during push-off phase were defined separately for each subject and condition (see supplemental material, Fig. S1). Vertical lines show approximate push-off phase for medium spring. Knee and hip power showed little variation within a group due to varying CESR spring stiffness. Amputees, however, seemed to exhibit large negative knee work (i.e., absorption) near terminal stance, which was not observed in non-amputees subjects. Amputees also appeared to perform more hip work during push-off as compared to non-amputees. Reference lines are shown for walking on a Conventional prosthesis (dashed line) and in street shoes (dotted line) for non-amputees.

ankle and COM push-off work. But there appeared to be biomechanical disadvantages to the softest spring (soft-PC), 
as intermediate stiffness (medium) yielded the best energetic economy. We also observed substantial differences in joint and COM work magnitudes between amputees and non-amputees, most notably during prosthetic limb push-off. These differences may provide insight into the effects of transtibial amputation on gait.

Regarding the energy-recycling spring, the general trend in both amputees and non-amputees was that the lower the spring stiffness, the greater the energy captured. Softer springs therefore led to more energy return, yielding higher prosthetic ankle push-off work, and ultimately to increased prosthetic limb COM push-off work. Pre-compression was necessary for the soft-PC spring due to the limited displacement range of the heel, but still allowed substantial energy storage, ${ }^{2}$ notably more than the medium or hard spring. Stiffer springs store and return less energy because they undergo less displacement for a given force. These trends do not, however, mean that the lowest stiffness is clinically optimal or yields the best overall function. In fact, the medium spring yielded the best energetic walking economy by $4 \%-8 \%$ in both groups, which may be relevant for amputees who typically expend about $10 \%-20 \%$ more energy than nonamputees [2], [3], [36], [37].

There are a number of possible disadvantages to the soft-PC and hard springs. First, storing too much energy in the soft-PC spring may have led to increased metabolic cost due to abnormally high COM energy losses during collision or due to interference with the natural Rebound of the body from earlyto mid- stance [Fig. 3(a)]. Meanwhile, the hard spring may not have stored and returned enough energy. A second disadvantage may be associated with high initial loading rates at heelstrike, due to the stiffness of the hard spring and pre-compression of the soft-PC spring. High loading rates may have caused discomfort or instability, leading subjects to walk with greater muscle co-contraction or other compensations that were metabolically costly. Further study may be needed to understand the effects of high initial loading rates due to pre-compression. However, the alternative was to not pre-compress the soft-PC spring, which we found to cause an even more uncomfortable collision when the spring fully compressed and the heel component bottomed out during gait. An alternative to pre-compression would be to increase the foot's internal range of motion to allow the soft spring to displace without bottoming out, but altering the range would affect the rolling shape of the foot and too large a range would lead to scuffing of the heel on the ground during leg swing. For the three springs tested in this study, rolling shapes were found to be relatively similar from mid- to late-stance, but did exhibit some qualitative differences during heelstrike collisions. ${ }^{7}$ Practical trade-offs between range of motion, energy storage and rolling shape are difficult to discern from gait analysis of three conditions, but this limitation does not change the main result: energy storage and return within the CESR foot increased with softening spring stiffness, but there were disadvantages to excessively low or high stiffness, such that some intermediate stiffness was optimal.

Although the CESR prototype was found to successfully return captured energy during push-off, the work may not have been performed optimally. Dynamic walking models predict

${ }^{7}$ See Figure S7 in supplementary downloadable material that push-off work of appropriate timing and magnitude can reduce collision work of the contralateral limb [10], [11] and thus reduce overall work on the COM. Such an effect was previously observed when comparing CESR with a conventional foot in non-amputees wearing a prosthetic simulator [17]. The negative trend between push-off and collision work magnitudes was also observed in other studies on amputees wearing conventional prostheses [9] and on non-amputees wearing shoes with different rolling shapes [38]. The trend was not, however, observed in this study when comparing across the three CESR springs, which yielded a smaller range of push-off magnitudes than the previous study [17]. Additionally, there are other studies that did not find that greater COM push-off work reduced collision work, such as when comparing typical non-amputee gait to gait with artificially fixed ankles [39] or when comparing impaired and unimpaired limbs [38]. In these cases, other factors may have played a more important role. For example, concomitant changes to limb mechanics may have dominated over the broad effect of push-off work on collision. Confounding factors such as timing of push-off, fluctuations in COM velocity, the peak power, or altered patterns of work production may have adversely affected the overall COM work magnitudes. In particular, we suspect that push-off should begin earlier in the gait cycle relative to collision, which is theoretically advantageous [10], [11] and for unilateral amputees, perhaps with magnitude more comparable to intact limb push-off.

Although the CESR foot performed similar amounts of energy storage and return in both groups, some COM and joint work measures varied substantially. Due to the various methodological differences, we had no a priori expectation that absolute COM and joint work magnitudes should be similar in amputees and non-amputees. However, since the CESR foot stored and returned approximately the same amount of energy in both groups, we were able observe how this similar magnitude of prosthesis work affected joint and COM mechanics. Notably, we found dramatic differences in prosthetic limb knee, hip and COM work during push-off. Despite a similar amount of CESR push-off work, amputees produced much less prosthetic limb COM push-off work than non-amputees, and much more knee and hip work during push-off (Fig. 5). In non-amputees, the magnitudes of prosthetic side knee and hip work were small and slightly positive during push-off. But in amputees, the substantial negative knee work appeared to partially absorb the CESR push-off work, reducing energy transfer from the prosthesis to the body COM and negating some of the benefits of prosthetic ankle push-off. Simultaneous positive power at the hip might then be a compensation for knee absorption in amputees and may lead to an increase in metabolic cost. Since inverse dynamics estimates cannot differentiate biarticular energy transfer from monarticular muscle work, we cannot directly estimate a metabolic penalty associated with this increased biological joint work. Overall, substantial kinetic differences between groups suggest amputees and non-amputees used prosthetic push-off differently.

In addition to push-off, we also observed qualitative differences in the collision phases of the intact and prosthetic limbs. Again, these differences are noteworthy given similar CESR foot function and energy storage in both groups (Fig. 2(b); 
Table I). Firstly, COM collision work done by both limbs appeared substantially lower in amputees than non-amputees (Fig. 3(d); Table III). Secondly, amputees performed little to no negative knee work during collision phase, while non-amputees performed significant negative knee work [Fig. 5(a)]. ${ }^{8}$ Thirdly, the rate of CESR spring loading after heelstrike appeared higher in non-amputees than amputees [Fig. 2(b)]. Collectively, these observations also suggest some differences in gait strategies used by amputees versus non-amputees. In general, amputees appeared to adapt collision strategies that resulted in smaller COM work magnitudes and less knee work. While these differences may be indicative of socket interface or comfort-related issues, it is interesting that they were also observed in the intact limb.

Although we observed similar trends in metabolic energy expenditure between the two groups, we do not quantitatively compare the absolute magnitudes. As with mechanical work, there was no reason to expect similar energetic magnitudes across groups given the differences between experimental protocols. Consequently, metabolic comparisons were only made within each group. Within group comparisons did suggest that metabolic rates for non-amputees were lower while walking on this CESR prototype than on the Conventional foot [17], but this relative trend was not observed in amputees. The CESR principle may still benefit amputee gait, although it may require slightly different design parameters or further user acclimation.

We propose several possible explanations for the observed mechanical and metabolic differences between amputees and non-amputees. The CESR foot may have been returning too much energy for the walking speed tested, such that amputees used knee absorption to regulate the excess power. This CESR prototype was originally designed for walking speeds faster than those studied in these groups (e.g., $1.5 \mathrm{~m} / \mathrm{s}$ ), when higher ankle/foot push-off is more desirable. Since amputees were walking more slowly, they may have adapted by overcompensating with negative knee work, effectively negating mechanical push-off benefits of the CESR foot. Another factor may have been that the CESR foot length was too long for the amputees. The prosthetic foot length relative to leg length was $11 \%$ longer in amputees than non-amputees due to the added height of the simulator boots. Longer feet tend to cause later push-off [12], which was observed in comparing amputees versus non-amputees [Fig. 3(a)], and may have led to associated changes in knee and hip kinetics. Another possibility may be that slower CESR spring loading (Fig. 2(b) inset) in amputees led to a suboptimal foot shape during rolling of the foot during single support. Rolling foot shape has been shown to affect metabolic cost substantially [12], [38]. Or amputees may adopt gait mechanics that are more heavily influenced by factors other than mechanical foot function. For example, the interface between the socket and residual limb may affect how comfortable it is for amputees to transmit forces between the prosthesis and the rest of the body, which may partially explain differences in knee and hip kinetics. Or the length of the residual limb may limit the ability to control energy storage and return in the foot, since shorter residual limbs have smaller moment arms to

${ }^{8}$ See Figures S3 and S5 in supplementary downloadable material generate torques and less surface area over which to distribute forces. Finally, a possible factor in this study could be related to insufficient acclimation time to the new CESR prosthesis. Despite similar adaptation times for amputees and non-amputees walking on the CESR foot (about $30 \mathrm{~min}$ ), amputees might have developed habits based on their prescribed passive prostheses that made it more difficult for them to adapt to a prosthesis with more energy storage and return. Or perhaps years of experience walking on Conventional feet biased amputees, who needed more time to adapt to the CESR foot. Such a bias would not be expected in naïve non-amputees, which may partially explain improved metabolic cost in this group.

We acknowledge several methodological limitations in directly comparing amputees to non-amputees. We tested amputees at slightly slower speed (1.14 versus $1.25 \mathrm{~m} / \mathrm{s})$ and with a less stiff hard spring (262 versus $324 \mathrm{~N} / \mathrm{mm}$ ). ${ }^{1}$ Experimental protocols were designed to be as identical as practical, but there were necessary differences because some amputee subjects had a slower comfortable walking speed on the treadmill and reported discomfort in the residual limb if the energy-recycling spring was too stiff. Additionally, amputees were tested at self-selected step frequency and non-amputees at a fixed metronome frequency, although resulting stride times were found to be nearly identical. ${ }^{9}$ We studied gait mechanics for amputees during overground walking, but for non-amputees during treadmill walking. Although kinematics and kinetics have been observed to be quite similar in overground versus treadmill walking in healthy individuals [40], [41], walking on a prosthesis may be more affected, perhaps due to balance-related issues that might be exacerbated on a treadmill. Given limited amputee subject availability and study time before fatigue onset, there were also practical limitations in how many different spring conditions could be tested. Three springs were selected to vary energy storage and return based on pilot studies performed on non-amputees.

Although prosthetic technologies are far from mature, it appears that specific mechanical parameters have deterministic effects on gait biomechanics. To understand these effects, it is necessary to conduct systematic studies that control or isolate parameters of interest. We found that systematically varying CESR spring stiffness led to altered prosthetic ankle mechanics, which in turn affected COM mechanics and metabolic energy expenditure. When measuring the same type of prosthesis with varying spring parameters, we found very similar trends in many mechanical and metabolic measures for amputees and non-amputees. We also found it helpful to perform comparisons with non-amputees wearing prosthetic simulators, since studying this group eliminates variability due to residual limb length, socket interface and secondary disabilities. Inter-group comparisons may provide insight regarding the disadvantages associated with the loss of an active ankle. Non-amputees exhibited substantial differences in work magnitudes for some COM and joint measures, suggesting that additional considerations may be important for optimizing prosthetic design parameters for amputees. Ultimately, more systematic studies of prosthetic design parameters could help inform fundamental design principles for feet.

${ }^{9}$ See Table S2 in supplementary downloadable material 


\section{REFERENCES}

[1] D. G. Barth, L. Schumacher, and S. S. Thomas, "Gait analysis and energy cost of below-knee amputees wearing six different prosthetic feet," JPO: J. Prosthetics Orthotics, vol. 4, no. 2, p. 63, 1992.

[2] L. M. Herbert, J. R. Engsberg, K. G. Tedford, and S. K. Grimston, “A comparison of oxygen consumption during walking between children with and without below-knee amputations," Physical Therapy, vol. 74, no. 10 , p. $943,1994$.

[3] R. S. Gailey et al., "Energy expenditure of trans-tibial amputees during ambulation at self-selected pace," Prosthetics Orthotics Int., vol. 18, no. 2, pp. 84-91, 1994.

[4] D. C. Norvell, J. M. Czerniecki, G. E. Reiber, C. Maynard, J. A. Pecoraro, and N. S. Weiss, "The prevalence of knee pain and symptomatic knee osteoarthritis among veteran traumatic amputees and nonamputees," Arch. Phys. Med. Rehabil., vol. 86, no. 3, pp. 487-493, 2005.

[5] P. A. Struyf, C. M. van Heugten, M. W. Hitters, and R. J. Smeets, "The prevalence of osteoarthritis of the intact hip and knee among traumatic leg amputees," Arch. Phys. Med. Rehabil., vol. 90, no. 3, pp. 440-446, 2009.

[6] D. M. Ehde, D. G. Smith, J. M. Czerniecki, K. M. Campbell, D. M. Malchow, and L. R. Robinson, "Back pain as a secondary disability in persons with lower limb amputations," Arch. Phys. Med. Rehabil., vol. 82, no. 6, pp. 731-734, 2001.

[7] D. C. Morgenroth, M. S. Orendurff, A. Shakir, A. Segal, J. Shofer, and J. M. Czerniecki, "The relationship between lumbar spine kinematics during gait and low-back pain in transfemoral amputees," Am. J. Phys. Med. Rehabil., vol. 89, no. 8, p. 635, 2010.

[8] J. M. Czerniecki, A. Gitter, and C. Munro, "Joint moment and muscle power output characteristics of below knee amputees during running: the influence of energy storing prosthetic feet," J. Biomechan., vol. 24, no. 1 , pp. 63-65, 1991.

[9] H. Houdijk, E. Pollmann, M. Groenewold, H. Wiggerts, and W. Polomski, "The energy cost for the step-to-step transition in amputee walking," Gait Posture, vol. 30, no. 1, p. 35, 2009.

[10] A. D. Kuo, "Energetics of actively powered locomotion using the simplest walking model," J. Biomechan. Eng., vol. 124, p. 113, 2002.

[11] A. Ruina, J. E. A. Bertram, and M. Srinivasan, "A collisional model of the energetic cost of support work qualitatively explains leg sequencing in walking and galloping, pseudo-elastic leg behavior in running and the walk-to-run transition," J. Theoretical Biol., vol. 237, no. 2, pp. 170-192, 2005.

[12] P. G. Adamczyk, S. H. Collins, and A. D. Kuo, "The advantages of a rolling foot in human walking," J. Exp. Biol., vol. 209, no. 20, p. 3953, 2006.

[13] D. A. Winter and S. E. Sienko, "Biomechanics of below-knee amputee gait," J. Biomechan., vol. 21, no. 5, pp. 361-367, 1988.

[14] A. Gitter, J. M. Czerniecki, and D. M. DeGroot, "Biomechanical analysis of the influence of prosthetic feet on below-knee amputee walking," Am. J. Phys. Med. Rehabil., vol. 70, no. 3, p. 142, 1991.

[15] D. C. Wing and D. A. Hittenberger, "Energy-storing prosthetic feet," Arch. Phys. Med. Rehabil., vol. 70, no. 4, p. 330, 1989.

[16] L. Torburn, J. Perry, E. Ayyappa, and S. L. Shanfield, "Below-knee amputee gait with dynamic elastic response prosthetic feet: A pilot study," J. Rehabil. Res. Develop., vol. 27, no. 4, pp. 369-84, 1990.

[17] S. H. Collins and A. D. Kuo, "Recycling energy to restore impaired ankle function during human walking," PLoS One, vol. 5, no. 2, p. E9307, 2010.

[18] S. Au, M. Berniker, and H. Herr, "Powered ankle-foot prosthesis to assist level-ground and stair-descent gaits," Neural Networks, vol. 21, no. 4, pp. 654-666, 2008.

[19] L. Torburn, C. M. Powers, R. Guiterrez, and J. Perry, "Energy expenditure during ambulation in dysvascular and traumatic below-knee amputees: A comparison of five prosthetic feet," Development, vol. 32, no. 2, pp. 111-119, 1995.

[20] J. F. Lehmann, R. Price, S. Boswell-Bessette, A. Dralle, K. Questad, and B. J. DeLateur, "Comprehensive analysis of energy storing prosthetic feet: Flex foot and Seattle foot versus standard sach foot," Arch. Phys. Med. Rehabil., vol. 74, no. 11, pp. 1225-1231, 1993.

[21] T. Schmalz, S. Blumentritt, and R. Jarasch, "Energy expenditure and biomechanical characteristics of lower limb amputee gait the influence of prosthetic alignment and different prosthetic components," Gait Posture, vol. 16, no. 3, pp. 255-263, 2002.
[22] K. Postema, H. J. Hermens, J. D. Vries, H. Koopman, and W. H. Eisma, "Energy storage and release of prosthetic feet Part 1: Biomechanical analysis related to user benefits," Prosthetics Orthotics Int., vol. 21, no. 1 , pp. 17-27, 1997.

[23] A. H. Hansen, M. R. Meier, P. H. Sessoms, and D. S. Childress, "The effects of prosthetic foot roll-over shape arc length on the gait of transtibial prosthesis users," Prosthetics Orthotics Int., vol. 30, no. 3, pp. 286-299, 2006

[24] E. Klodd, A. Hansen, S. Fatone, and M. Edwards, "Effects of prosthetic foot forefoot flexibility on oxygen cost and subjective preference rankings of unilateral transtibial prosthesis users," J. Rehabil. Res. Develop., vol. 47, no. 6, pp. 543-552, 2010.

[25] C. L. Johnson and E. L. Robinson, "Able-bodied artificial foot phosthesis testing apparatus," U.S. 6238437, May 2001.

[26] A. H. Hansen, D. S. Childress, and E. H. Knox, "Prosthetic foot rollover shapes with implications for alignment of trans-tibial prostheses," Prosthetics Orthotics Int., vol. 24, no. 3, pp. 205-215, 2000.

[27] D. L. Poggi, E. M. Burgess, D. E. Moeller, and D. A. Hittenberger, "Prosthetic foot having a cantilever spring keel," U.S. 4645509, 1987.

[28] J. M. Donelan, R. Kram, and A. D. Kuo, "Simultaneous positive and negative external mechanical work in human walking," J. Biomechan., vol. 35 , no. 1 , pp. 117-124, 2002.

[29] D. G. Robertson and D. A. Winter, "Mechanical energy generation, absorption and transfer amongst segments during walking," $J$. Biomechan., vol. 13 , no. 10 , p. $845,1980$.

[30] S. Y. Aleshinsky, “An energy sources' and fractions' approach to the mechanical energy expenditure problem-I. basic concepts, description of the model, analysis of a one-link system movement," J. Biomechan., vol. 19, no. 4, pp. 287-293, 1986.

[31] F. Prince, D. A. Winter, G. Sjonnesen, and R. K. Wheeldon, "A new technique for the calculation of the energy stored, dissipated, and recovered in different ankle-foot prostheses," IEEE Trans. Rehabil. Eng., vol. 2, no. 4, pp. 247-255, Dec. 1994

[32] D. A. Winter, The Biomechanics and Motor Control of Human Gait: Normal, Elderly and Pathological. Waterloo, Canada: Univ. Waterloo Press, 1991.

[33] J. M. Donelan, R. Kram, and A. D. Kuo, "Mechanical work for step-tostep transitions is a major determinant of the metabolic cost of human walking," J. Exp. Biol., vol. 205, no. 23, pp. 3717-3727, 2002.

[34] A. D. Kuo, J. M. Donelan, and A. Ruina, "Energetic consequences of walking like an inverted pendulum: Step-to-step transitions," Exercise Sport Sci. Rev., vol. 33, no. 2, p. 88, 2005.

[35] J. M. Brockway, "Derivation of formulae used to calculate energy expenditure in man," Human Nutrition Clin. Nutrition, vol. 41, no. 6, pp. 463-71, 1987.

[36] N. H. Molen, "Energy/speed relation of below-knee amputees walking on a motor-driven treadmill," Eur. J. Appl. Physiol. Occupat. Physiol., vol. 31, no. 3, pp. 173-185, 1973.

[37] J. J. Genin, G. J. Bastien, B. Franck, C. Detrembleur, and P. A. Willems, "Effect of speed on the energy cost of walking in unilateral traumatic lower limb amputees," Eur. J. Appl. Physiol., vol. 103, no. 6, pp. 655-663, 2008.

[38] S. J. P. M. van Engelen, Q. E. Wajer, L. W. van der Plaat, H. C. Doets, C. N. van Dijk, and H. Houdijk, "Metabolic cost and mechanical work during walking after tibiotalar arthrodesis and the influence of footwear," Clin. Biomechan. (Bristol, Avon), vol. 25, no. 8, pp. 809-815, Oct. 2010.

[39] M. T. Vanderpool, S. H. Collins, and A. D. Kuo, "Ankle fixation need not increase the energetic cost of human walking," Gait \& posture, vol. 28 , no. 3 , p. 427,2008

[40] S. J. Lee and J. Hidler, "Biomechanics of overground vs. Treadmill walking in healthy individuals," J. Appl. Physiol., vol. 104, no. 3, pp. 747-755, Mar. 2008.

[41] P. O. Riley, G. Paolini, U. D. Croce, and K. W. Kerrigan, "A kinematic and kinetic comparison of overground and treadmill walking in healthy subjects," Gait Posture, vol. 26, no. 1, pp. 17-24, 2007.

[42] T. McGeer, "Passive dynamic walking," Int. J. Robot. Res., vol. 9, no. 2, p. 62, 1990.

Authors' photographs and biographies not available at the time of publication. 